\title{
Prognostic and Clinicopathologic Significance of Discoidin Domain Receptors in Different Human Malignancies: A Meta-Analysis
}

\author{
Gordon A. Ferns ${ }^{a}$ Sheida Shabanian ${ }^{b}$ Milad Shahini Shams Abadi ${ }^{c}$ \\ Ahmadshah Farhat $^{d}$ Mohammad-Hassan Arjmand ${ }^{e, f}$
}

aDivision of Medical Education, Brighton \& Sussex Medical School, Brighton, UK; ${ }^{b}$ Department of Obstetrics and Gynecology, Faculty of Medicine, Shahrekord University of Medical Sciences, Shahrekord, Iran; 'Department of Microbiology and Immunology, Cellular and Molecular Research Center, Basic Health Sciences Institute, Shahrekord University of Medical Sciences, Shahrekord, Iran; ${ }^{d}$ Neonatal Research Center, Emam Reza Hospital, Mashhad University of Medical Sciences, Mashhad, Iran; 'Cancer Research Center, Shahrekord University of Medical Sciences, Shahrekord, Iran; ${ }^{\mathrm{M}}$ Medical Plants Research Center, Basic Health Sciences Institute, Shahrekord University of Medical Sciences, Shahrekord, Iran

\section{Keywords}

Discoidin domain receptors . Cancer prognosis .

Meta-analysis

\begin{abstract}
Background: Discoidin domain receptors (DDRs) belong to the receptor tyrosine kinases family and are activated by different types of collagens, which play roles in various physiological processes. An abnormal expression of DDRs is reported in different types of cancers. Despite many reports about the association and roles of high DDR expression levels in cancers, the prognostic values of DDRs are still unclear. This meta-analysis was performed to evaluate the prognostic effect of DDRs in different tissue cancers. Method: A literature search was performed in several related databases to find eligible English articles. Based on our research, 20 appropriate studies with 2,602 patients were selected till October 5,2020 . The pooled hazard ratio (HR) with a corresponding $95 \%$ confidence interval $(\mathrm{Cl})$ was computed to evaluate the strength of correlation between DDRs and survival of cancer patients. Result: Pooling results showed that a high
\end{abstract}

karger@karger.com www.karger.com/gat

Karger"

BOPEN ACCESS
(C) 2021 The Author(s)

Published by S. Karger AG, Basel

This is an Open Access article licensed under the Creative Commons Attribution-NonCommercial-4.0 International License (CC BY-NC) (http://www.karger.com/Services/OpenAccessLicense), applicable to the online version of the article only. Usage and distribution for commercial purposes requires written permission.
DDR expression was significantly associated with poorer overall survival (OS) $(\mathrm{HR}=1.304,95 \% \mathrm{Cl} 1.007-1.69, p=0.04)$. Subgroup analysis based on cancer type revealed a significant link between a high DDR expression level and poor OS both in gastrointestinal (pooled $\mathrm{HR}=1.78,95 \% \mathrm{Cl} 1.214$ 2.624, $p=0.003$ ) and urological cancers (pooled $\mathrm{HR}=1.42$, $95 \% \mathrm{Cl} 1.062-1.82, p=0.018)$. Conclusion: Our meta-analysis results suggest that high DDRs expression has the potential to be used as a biomarker of poor prognosis in cancers.

(c) 2021 The Author(s)

Published by S. Karger AG, Basel

\section{Introduction}

Cancer is characterized by an abnormal proliferation of cells and with the ability to metastasize and invades different parts of the body. The rising trend of cancers is a big concern globally. According to this, approximately about 1,762,450 new cases related to cancers were diagnosed, and 606,880 death occurred in the USA in 2019 [1]. The assessment of cancer prognosis plays an important role in oncology and includes the prediction of pa- 
tient's survival and guide therapy [2]. Despite the recent development of multidisciplinary synthetic therapy, the prognosis of patients with late stages of malignant tumors remains unsatisfactory [3]. To this end, developing new specific biomarkers for cancer prognosis has great clinical value in the follow-up of patients which can be applied to targeted therapy of tumors and promote patient's survival.

Discoidin domain receptors (DDRs) are a family of receptor tyrosine kinases (RTKs) that are responsible for the response of collagen and an appealing anti-fibrotic target $[1,2]$. These receptors are composed of 2 types, $\mathrm{DDR}_{1}$ and $\mathrm{DDR}_{2}$, which contain a discoidin homology domain in the extracellular region for collagen binding, then undergo autophosphorylation of intracellular catalytic domains to mediate cellular response [2], although there are limited studies that describe the signaling pathways stimulated by DDRs upon collagen attachment. DDRs are distributed in different organs; in solid tissues, $D_{D R}$ is more expressed in epithelial cells, and $D_{D R}$ is limited to mesenchymal cells [4]. Recently, studies have shown that the expression of DDRs upregulated in different types of malignancies such as hepatocellular carcinoma (HCC) [5, 6], gastric cancer [4], ovarian cancer $[5,7$, $8]$, lung cancer $[6,9,10]$, pancreatic carcinoma [11], and breast cancer $[12,13]$. To the best of our knowledge, no systematic review and meta-analysis has been done to assess the relation between DDR expression and clinicopathologic features and prognostic value of DDRs in patients with different malignancies; so in this meta-analysis, we systematically pooled related published evidence to explain the prognostic significance of DDR expression in malignant tumors.

\section{Methods}

The study was conducted and described according to the Preferred Reporting Items for Systematic Reviews and Meta-Analyses (PRISMA) statement.

\section{Literature Search Strategy}

An electronic literature search was systematically carried out in databases including PubMed, Web of Science, Scopus, Google Scholar, and Embase to get pertinent English publication with reference to prognostic and clinicopathologic features of DDRs in malignant tumors up to October 5, 2020. The searched keywords included ("Discoidin Domain Receptor" or "DDR Proteins") AND ("cancer" or "neoplasm" or "malignancy" or "tumor" or "carcinoma") AND ("prognostic factor" or "prognosis") were used. Also, we checked reference lists in selected articles to assess the potential of the related article and increase the accuracy of the search process.

\section{Inclusion and Exclusion Criteria}

The inclusion criteria for our study were as follows: (1) evaluation of the expression levels of DDRs ( 1 or 2 ) in malignant tissue, (2) divided the patients into 2 groups regarding the DDRs expression levels, (3) adequate data for estimation of the hazard ratio (HR) with $95 \%$ confidence interval (CI) and the correlation between survival and DDR expression, and (4) the association between clinicopathologic features and prognostic information. The exclusion criteria were as follows: (1) unpublished studies, (2) articles such as review, case report, letter to editor, and animal studies or articles with inadequate or unavailable data, (3) overlapping articles or duplicate data, and (4) non-English articles.

\section{Data Extraction}

Two independent investigators (Mohammad-Hassan Arjmand and Milad Shahini Shams Abadi) carried out data extraction from all appropriate publications. Any disagreement was resolved through consulting with a third author (Sheida Shabanian). The following information was recorded from each study including first author name, publication year, region, cancer type, sample size, source of DDR, DDR isoform, and DDR detection assay. Moreover clinicopathologic parameters such as gender, tumor size, tumor stage, lymph node metastasis (LNM), distance metastasis, and HR with its $95 \%$ CI for overall survival (OS) were collected. The quality assessment of included articles was evaluated according to the Newcastle-Ottawa Scale (NOS) [14] by 2 authors independently (M.-H.A. and M.S.S.A.). A score with higher or equal to 6 points could be reflected as high quality (Table 1). If the HR and 95\% CI were not directly reported and only Kaplan-Meier curves were shown in some articles, the HR and 95\% CI were calculated as reported by Parmar's formula [15].

\section{Statistical Analysis}

All analyses were performed using Comprehensive MetaAnalysis (Biostat, Englewood, NJ, USA), a computer program. The $p$ value $<0.05$ showed that the result had a statistical significance. The effects of DDR expression on the OS of patients in various cancers were reported as HRs with 95\% CIs. The association between DDR expression and clinicopathologic parameters was considered by odd ratios (ORs) and corresponding 95\% CIs. The heterogeneity was evaluated by Cochrane's $Q$ test and the $I^{2}$ index among different studies. If $I^{2}>50 \%$ and $p<0.05$ among studies, the random-effects model was selected. Otherwise, the fixed-effects model was applied. The publication bias was measured by using a funnel plot and Egger's linear regression test. Sensitivity analysis was also performed to consider the stability of the collected results.

\section{Results}

\section{Literature Search and Data Characteristics}

A total of 146 relevant studies were identified from electronic databases. After assessment of titles and abstracts, 105 studies were removed because they were basic research, reviews, animal studies, case report, or duplicate articles. After that, the remaining 41 studies were read completely, and 21 articles were excluded due to inade- 


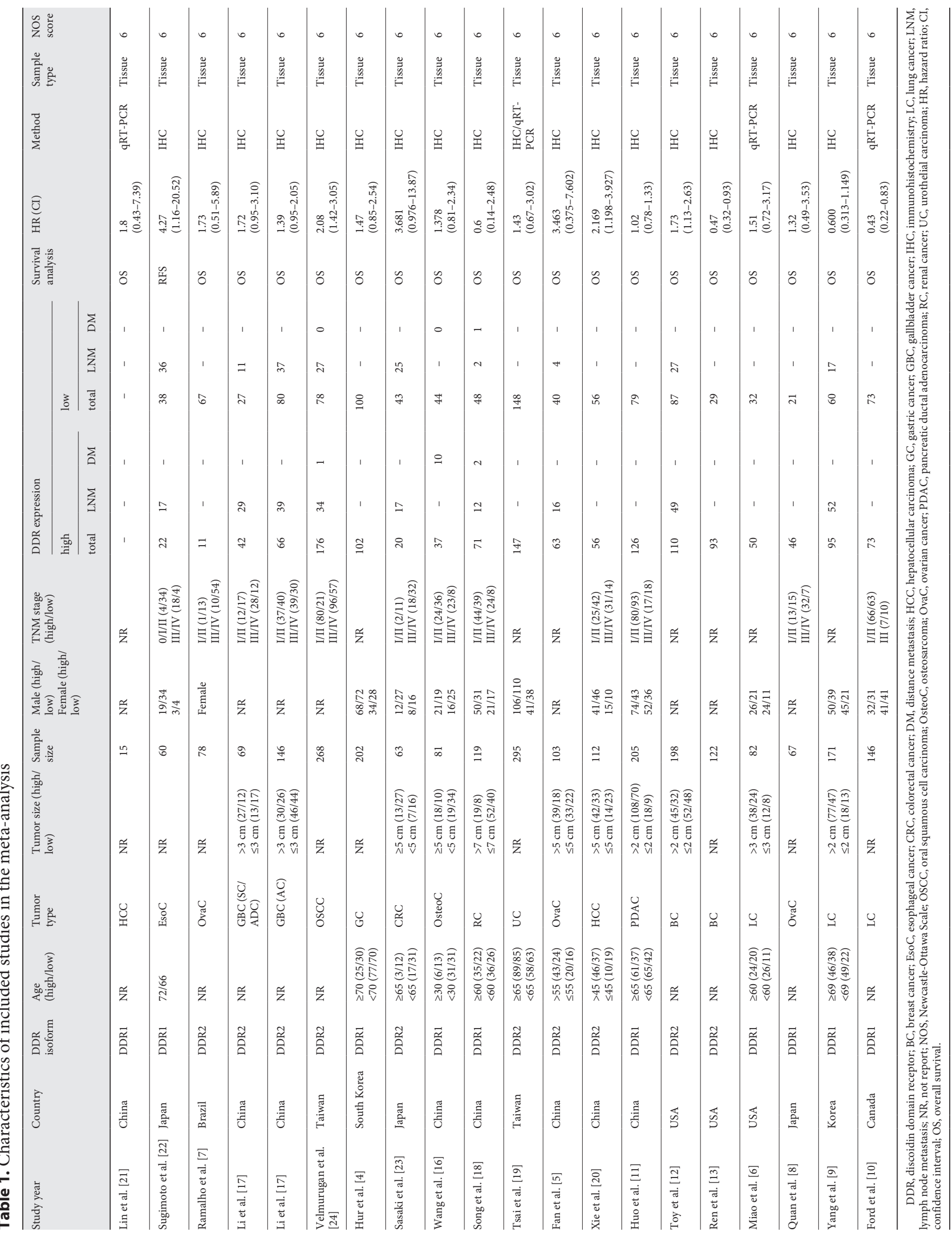


quate data to evaluate the HR for quantitative analysis. Ultimately, 20 appropriate studies with the inclusion criteria were selected showing agreement for inclusion in the meta-analysis. The process of study selection is abstracted in Figure 1. The general characteristics of the research studies involved in the analysis from 2007 to 2020 are summarized in Table 1.

In total, there were 2,602 patients. Also, the majority of involved studies were reported by authors in Asia (China, Japan, Taiwan, and South Korea), and the other studies were 1 from Canada, 3 from the USA, and 1 from Brazil. Moreover, 12 types of cancers including 3 ovarian cancers [5,7,9], 2 breast cancers [12, 13], 1 osteosarcoma [16], 3 lung cancers $[6,9,10]$, urological cancers (UC) which include 2 gallbladder carcinomas [17], 1 renal cancer [18], and 1 urothelial carcinoma [19], and gastrointestinal (GI) malignancies which include 1 gastric cancer [4], 2 HCCs [20, 21], 1 esophageal cancer [22], 1 colorectal cancer [23], 1 pancreatic carcinoma [11], and 1 oral cell carcinoma [24] were evaluated in this meta-analysis. The total subjects registered were divided into high and low DDR groups based on the DDR measurement results.

According to the HR estimations, the HR values were directly described from 12 studies, while for 8 studies, the HRs were calculated through data reading from KaplanMeier survival curves. In relation to clinicopathologic parameters, 9 articles provided data according to the association between DDR expression and LNM, 12 articles reported TNM stage, 11 articles reported tumor size, and 10 articles evaluated age.

\section{Association between DDR Expression and Survival in Cancer}

As for survival analysis, a total of 20 studies (a total number of patients $=2,602$ ) had calculated the relation of DDR expression with OS. A random-effects framework was applied because of the significant heterogeneity among these studies $\left(I^{2}=70.6 \%, p=<0.001\right)$. The pooled HR showed that high expression levels of DDRs (DDR and $\mathrm{DDR}_{2}$ ) were significantly correlated with poor $\mathrm{OS}$ compared with the low DDR expression (pooled HR = $1.304,95 \%$ CI 1.007-1.69, $p=0.04$ ) (Fig. 2). In addition, subgroup analyses were done according to cancer type (GI cancers, UC, and other malignancies), sample size, ethnicity, and DDR isoform (Table 2). The classification analysis by cancer types showed a significant link between a high DDR expression level and poor OS both in UC (pooled HR $=1.42,95 \%$ CI 1.062-1.82, $p=0.018$ ) and GI cancers $($ pooled $\mathrm{HR}=1.78,95 \%$ CI 1.214-2.624, $p=$ 0.003 ) but not significant in other cancers (pooled HR =

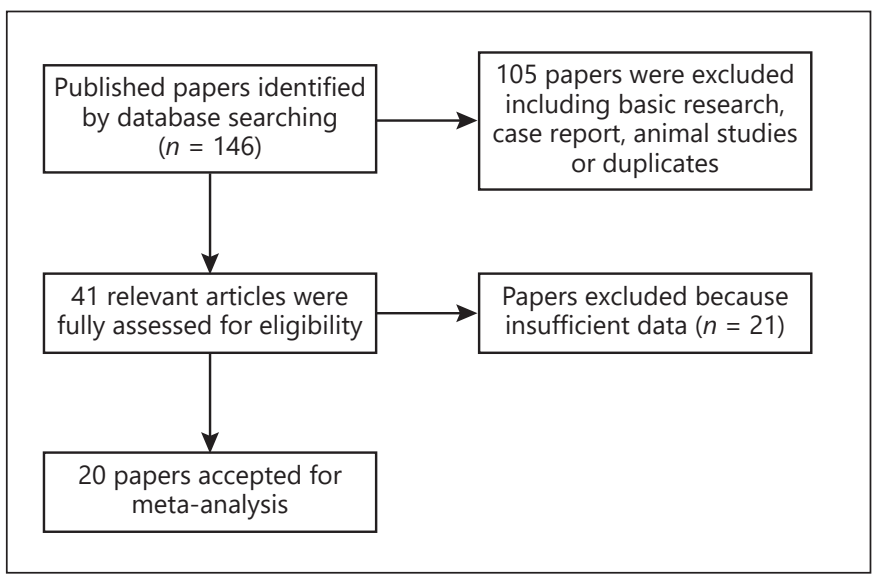

Fig. 1. Flow diagram of the study selection.

1.002, 95\% CI 0.631-1.59, $p=0.99$ ) (Fig. 3). In subgroup analysis according to sample size, a significant association was observed between DDR upregulation and OS in sample sizes $<100$ (pooled HR $=1.676,95 \%$ CI 1.250-2.24, $p=0.001)$. However, there was no significant association between DDR overexpression and sample size $\geq 100$ (pooled HR $=1.119,95 \%$ CI 0.798-1.570, $p=0.51)(\mathrm{Ta}-$ ble 2). In addition, high DDR expression was significantly related to poor OS in the Asian population based on ethnicity (pooled HR $=1.358,95 \%$ CI 1.051-1.754, $p=$ 0.019 ) (Table 2). Finally, subgroup analysis based on the DDR isoform showed that just there was a significant association between DDR2 and poor OS (pooled $\mathrm{HR}=1.55$ 95\% CI 1.035-2.330, $p=0.034$ ) against DDR1 expression (pooled HR $=1.8495 \%$ CI 0.788-1.491, $p=0.62$ ).

\section{Correlation between DDRs and Clinicopathologic \\ Characteristics}

A meta-analysis was performed to evaluate the association between the DDR expression level and clinicopathologic features. The pooled OR and $95 \%$ CI of all outcomes including gender, age, LNM, DM, tumor size, and TNM stage are presented in Table 3. Reports from a collection of 11 studies found that the association of DDRs with gender in different tumors (pooled $\mathrm{OR}=2.86,95 \%$ CI 1.637-5.01, $p<0.001, I^{2}=85.02 \% p<0.001$, randomeffects model) (Fig. 4) (Table 3). Nevertheless, no significance association was observed between overexpression of DDRs with TNM stage (pooled OR $=1.642,95 \%$ CI $0.508-5.309, p=0.4, I^{2}=95.6 \% p<0.001$, random-effects model), tumor size (pooled OR $=0.395,95 \%$ CI 0.128 $1.218, p=0.1, I^{2}=95.6 \% p<0.001$, random-effects model), LNM (pooled OR $=0.989,95 \%$ CI $0.263-3.718, p=$ 


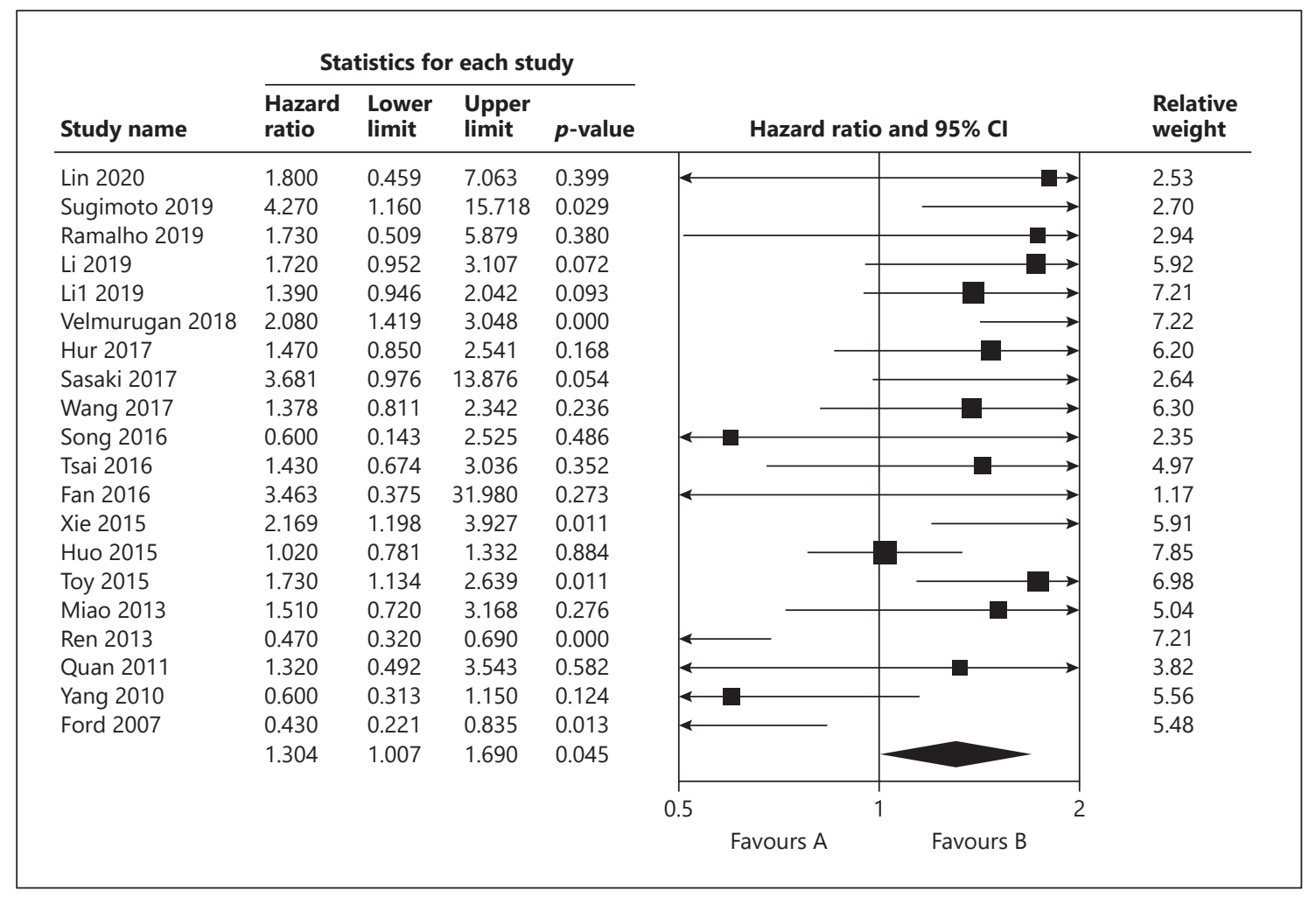

Fig. 2. Forest plots for the association between SNHG20 expression and OS; HR with 95\% CI. Pooled HR shows the association between OS and DDR expression on various cancers. OS, overall survival; HR, hazard ratio; CI, confidence interval; DDR, discoidin domain receptor.

Table 2. Stratified analyses of pooled HRs for OS

\begin{tabular}{|c|c|c|c|c|c|c|c|}
\hline \multirow[t]{2}{*}{ Category } & \multirow{2}{*}{$\begin{array}{l}\text { Studies, } \\
n\end{array}$} & \multirow{2}{*}{$\begin{array}{l}\text { Patients, } \\
n\end{array}$} & \multicolumn{2}{|l|}{ Test of association } & \multicolumn{3}{|c|}{ Test of heterogeneity } \\
\hline & & & pooled HR (95\% CI) & $p$ value & $I^{2}, \%$ & $p$ value & Model \\
\hline \multicolumn{8}{|l|}{ Cancer type } \\
\hline GI & 7 & 924 & $1.78(1.214-2.624)$ & 0.003 & 63.6 & 0.011 & $\mathrm{R}$ \\
\hline UC & 4 & 629 & $1.42(1.062-1.82)$ & 0.0018 & 76.3 & $<0.001$ & $\mathrm{R}$ \\
\hline Others & 8 & 1,049 & $1.002(0.631-1.59)$ & 0.99 & 0 & 0.6 & $\mathrm{R}$ \\
\hline \multicolumn{8}{|l|}{ Sample size } \\
\hline$\geq 100$ & 12 & 2.087 & $1.119(0.798-1.570)$ & 0.51 & 79.9 & $<0.001$ & $\mathrm{R}$ \\
\hline$<100$ & 8 & 515 & $1.676(1.250-2.24)$ & 0.001 & 0 & 0.76 & $\mathrm{~F}$ \\
\hline \multicolumn{8}{|l|}{ Ethnicity } \\
\hline Asian & 16 & 2,122 & $1.358(1.051-1.754)$ & 0.019 & 86.9 & $<0.001$ & $\mathrm{R}$ \\
\hline American & 4 & 480 & $1.149(0.51-2.58)$ & 0.73 & 59.2 & 0.001 & $\mathrm{R}$ \\
\hline \multicolumn{8}{|l|}{ DDR isoform } \\
\hline DDR1 & 10 & 1,148 & $1.84(0.788-1.491)$ & 0.62 & 52.77 & 0.025 & $\mathrm{R}$ \\
\hline DDR2 & 10 & 1,454 & $1.55(1.035-2.330)$ & 0.034 & 78.2 & $<0.001$ & $\mathrm{R}$ \\
\hline
\end{tabular}

DDR, discoidin domain receptor; UC, urological cancer; GI, gastrointestinal; OS, overall survival; HR, hazard ratio; CI, confidence interval. 


\begin{tabular}{|c|c|c|c|c|c|c|c|}
\hline \multirow[b]{2}{*}{$\begin{array}{l}\text { Group by } \\
\text { cancer type }\end{array}$} & \multirow[b]{2}{*}{ Study name } & \multicolumn{4}{|c|}{ Statistics for each study } & \multirow{2}{*}{\multicolumn{2}{|c|}{ Hazard ratio and $95 \% \mathrm{Cl}$}} \\
\hline & & $\begin{array}{l}\text { Hazard } \\
\text { ratio }\end{array}$ & $\begin{array}{l}\text { Lower } \\
\text { limit }\end{array}$ & $\begin{array}{l}\text { Upper } \\
\text { limit }\end{array}$ & $p$-value & & \\
\hline Gl cancer & Lin 2020 & 1.800 & 0.459 & 7.063 & 0.399 & & \\
\hline Gl cancer & Sugimoto 2019 & 4.270 & 1.160 & 15.718 & 0.029 & & \\
\hline GI cancer & Velmurugan 2018 & 2.080 & 1.419 & 3.048 & 0.000 & & \\
\hline Gl cancer & Hur 2017 & 1.470 & 0.850 & 2.541 & 0.168 & & \\
\hline GI cancer & Sasaki 2017 & 3.881 & 0.976 & 13.876 & 0.054 & & \\
\hline Gl cancer & Xie 2015 & 2.169 & 1.198 & 3.927 & 0.011 & & \\
\hline Gl cancer & Huo 2015 & 1.020 & 0.781 & 1.332 & 0.884 & & - \\
\hline Gl cancer & & 1.785 & 1.214 & 2.624 & 0.003 & & \\
\hline Other & Ramalho 2019 & 1.730 & 0.509 & 5.879 & 0.380 & & \\
\hline Other & Wang 2017 & 1.378 & 0.811 & 2.342 & 0.238 & & \\
\hline Other & Fan 2018 & 3.403 & 0.375 & 31.980 & 0.273 & $\leftarrow$ & \\
\hline Other & Toy 2015 & 1.730 & 1.134 & 2.639 & 0.011 & & \\
\hline Other & Miao 2013 & 1.510 & 0.720 & 3.168 & 0.276 & & \\
\hline Other & Ren 2013 & 0.470 & 0.320 & 0.690 & 0.000 & $\leftarrow$ & \\
\hline Other & Guan 2011 & 1.320 & 0.492 & 3.543 & 0.582 & $\leftarrow$ & \\
\hline Other & Yang 2010 & 0.600 & 0.313 & 1.150 & 0.124 & $\leftarrow$ & \\
\hline Other & Ford 2007 & 0.430 & 0.221 & 0.835 & 0.013 & $\leftarrow$ & \\
\hline Other & & 1.002 & 0.631 & 1.593 & 0.993 & & \\
\hline UC & Li 2019 & 1.720 & 0.952 & 3.107 & 0.072 & & \\
\hline UC & Li1 2019 & 1.390 & 0.946 & 2.042 & 0.093 & & \\
\hline UC & Song 2018 & 0.600 & 0.143 & 2.525 & 0.486 & & \\
\hline UC & Tsai 2016 & 1.430 & 0.674 & 3.036 & 0.352 & & \\
\hline UC & & 1.420 & 1.062 & 1.898 & 0.018 & & \\
\hline \multirow[t]{3}{*}{ Overall } & & 1.415 & 1.150 & 1.741 & 0.001 & & \\
\hline & & & & & & 0.5 & 1 \\
\hline & & & & & & Favours A & 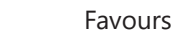 \\
\hline
\end{tabular}

Fig. 3. Forest plot based on subgroup analysis showed a significant association between high DDR expression and poor OS in UC and GI cancers. UC, urological cancer; GI, gastrointestinal; OS, overall survival; DDR, discoidin domain receptor.

Table 3. Meta-analysis of the association between DDR expression and clinicopathologic characteristics

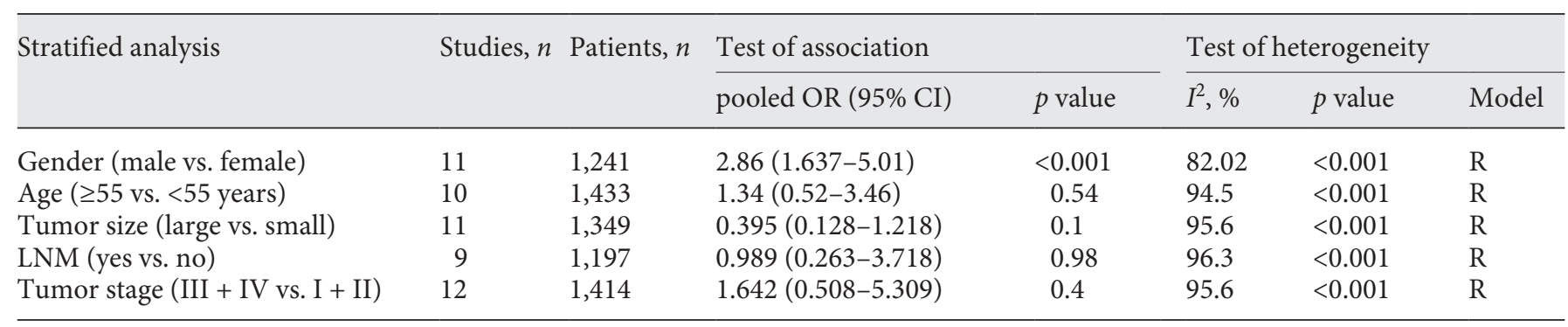

DDR, discoidin domain receptor; OR, odds ratio; CI, confidence interval; LNM, lymph node metastasis.

$0.98, I^{2}=96.3 \% p<0.001$, random-effects model), and age (pooled OR $=1.34,95 \%$ CI $0.52-3.46, p=0.54, I^{2}=94.5 \%$ $p<0.001$, random-effects model) on patients (Table 3 ).

\section{Sensitivity Analysis}

Sensitivity analysis was conducted to evaluate the effect of each study on the robustness of the analysis. In our meta-analysis, the pooled HR was not significantly influenced by any single study (Fig. 5).

\section{Publication Bias}

Begg's test and Egger's test were also carried out to evaluate the publication bias for the present meta-analysis. The outcome of Begg's test $(p=0.97)$ and Egger's test 
Fig. 4. Forests plots for the association between DDR expression and gender; OR with 95\% CI. DDR, discoidin domain receptor; OR, odds ratio; $\mathrm{CI}$, confidence interval.

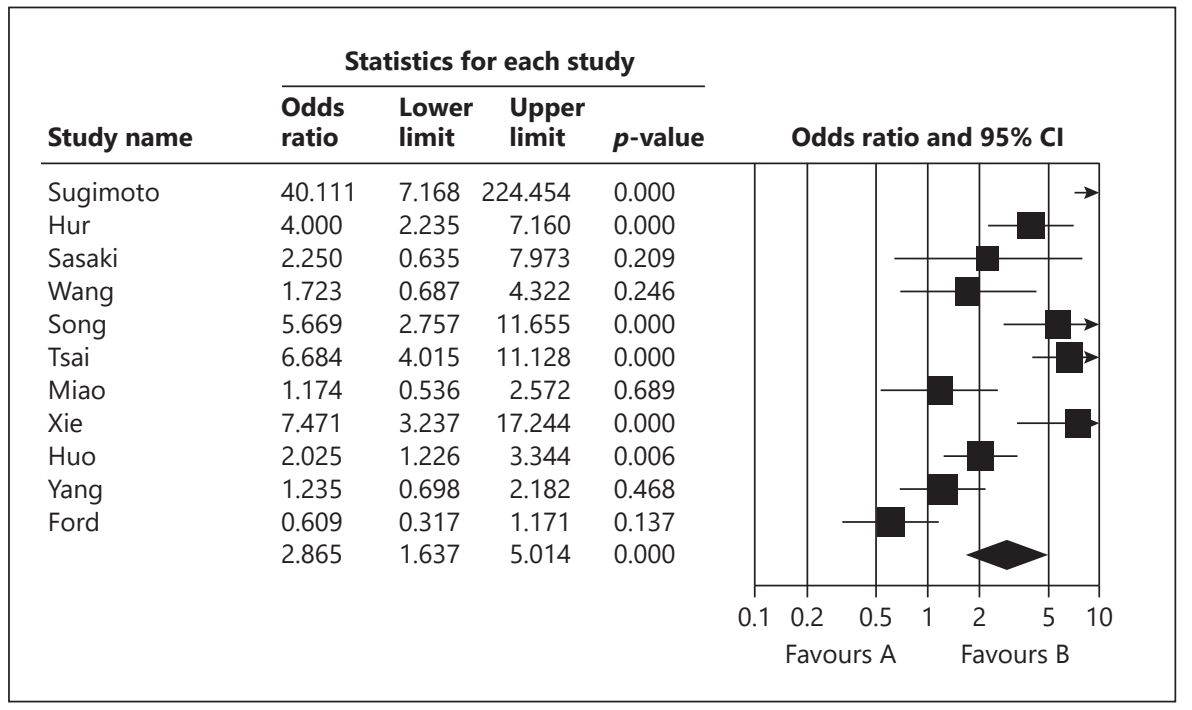

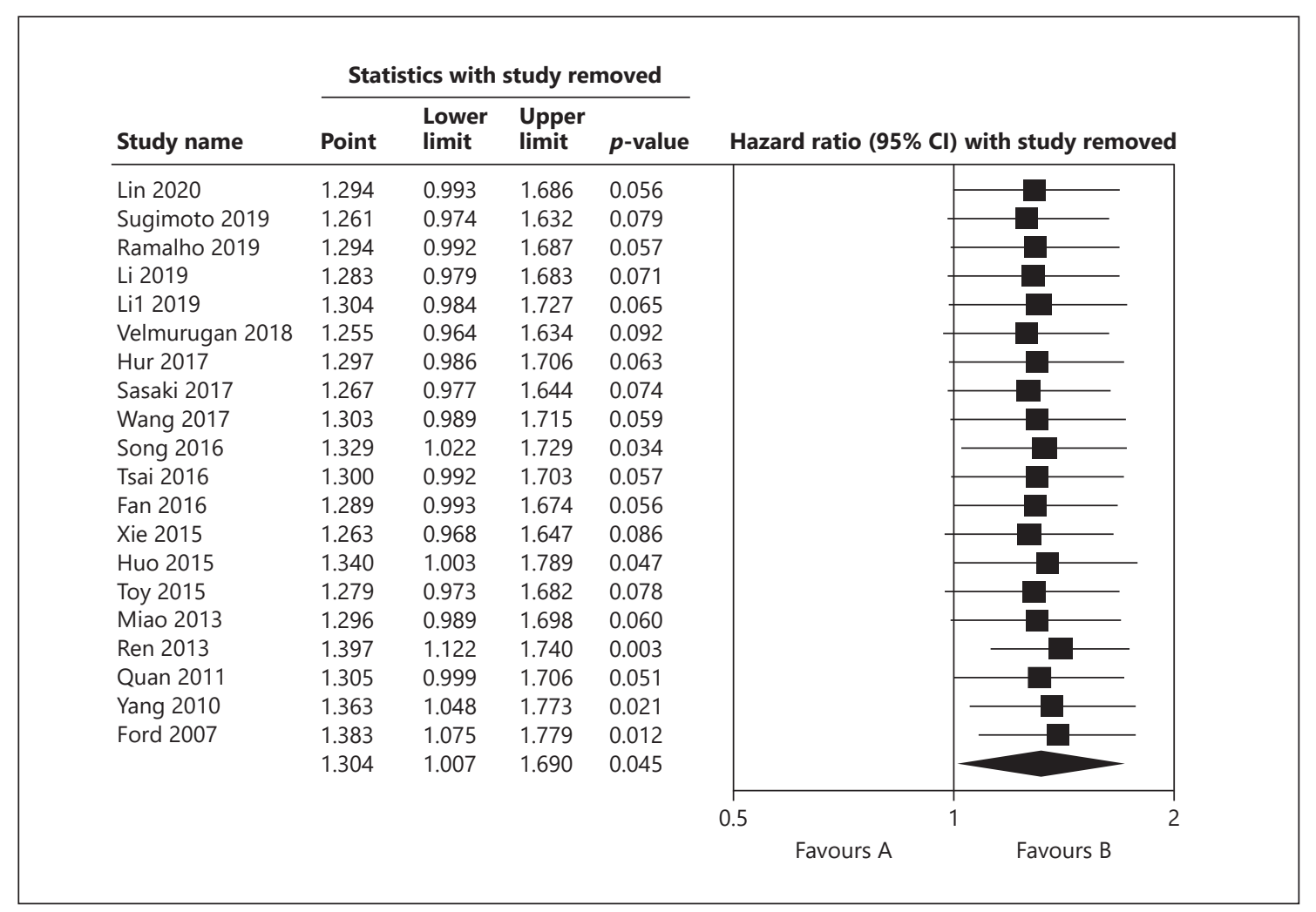

Fig. 5. Sensitivity analysis for the correlation between DDR expression with OS. DDR, discoidin domain receptor; OS, overall survival.

$(p=0.31)$ results for OS conducted that there was no significant publication bias across the included studies. Furthermore, there was no publication bias based on Egger's test results for ORs of DDR overexpression on gender, age, tumor size, TNM stage, and LNM.

Discoidin Domain Receptors and Cancer Prognosis

\section{Discussion}

Although there have been advances in cancer prediction and treatment during the past decades, many cancers cannot currently be adequately treated because of 
the lack of efficient biomarkers for early detection and subsequent useful treatment at the terminal stages. Currently, many investigations have concentrated on finding tumor markers to predict cancer prognosis. Now, increasing evidence supports the association between DDRs and collagen overexpression and cancer risk. In this way, several investigations have described altered DDRs expression in different types of cancer $[4,6,19]$. In the current study, we showed the correlation between DDR expression and worse overall prognosis in malignant tumors in patients.

Although there are limited data on the role of DDRs and their molecular targets, they have been suggested to be essential to control cell behavior. DDRs and tyrosine kinases have been shown to be included in a wide range of cell functions such as cellular proliferation, differentiation, migration, cytokines secretion, and extracellular matrix (ECM) hemostasis and remodeling [25]. Triple-helical conformations of collagens are the main ligands for both receptors of DDR. Different types of collagen (mainly type 1) can active DDRs [26, 27]. While activation of DDRs is needed for normal cell function, studies have demonstrated the upregulation and mutations of $\mathrm{DDR}_{1}$ and $\mathrm{DDR}_{2}$ in different cancers [25]. The emerging role of DDRs in the proliferation and survival of tumor cells and their association with oncogenic signaling are shown in in vitro and in vivo studies $[28,29]$. Investigation in human colon carcinoma cell lines (HCT116) indicated that $\mathrm{DDR}_{1}$, in response to collagen-induced activation, stimulates cell survival through activation of Notch signaling [30]. Another study showed that overexpression of $\mathrm{DDR}_{2}$ is the result of mutations and promotes cell growth and proliferation in NIH3T3 mouse fibroblast cells [31]. Han et al. [32] have reported that $\mathrm{DDR}_{2}$ has the potential to upregulate cell growth and proliferation of human osteosarcoma cell lines by overexpression of cyclooxygenase 2. Epithelial-mesenchymal transition (EMT) contributes to fibrosis and tumor progression through different mechanisms. Tumor cells by expression of epithelial and mesenchymal markers promote tumor migration to other organs. In relation to this, $D_{D R}$ is an epithelial marker, and $\mathrm{DDR}_{2}$ is a mesenchymal marker besides well-known EMT markers such as vimentin and $\mathrm{N}$-cadherin. Therefore, overexpression of $\mathrm{DDR}_{1}$ and $\mathrm{DDR}_{2}$ reflects a result of the EMT process toward the majority of malignant tumor cells [12,33]. DDR1 and DDR2 can support EMT and so have the potential to contribute to tumor cell migration. Herein, various studies reported the role of $\mathrm{DDR}_{1}$ in the regula- tion of cell migration in different malignant cell lines such as HCC, pancreas, breast, colorectal cancer, and lung $[9,29,34,35]$. In addition, tumor cell invasion is a complicated process performed by cancer cells to attack to other organs. Cell invasion requires ECM degradation and tissue remodeling. Some reports indicated that $\mathrm{DDR}_{1}$ can induce the matrix metalloproteinase 2 (MMP2) and MMP9, which play an important role in ECM degradation $[6,36]$. Hu et al. [37] reported that overexpression of $\mathrm{DDR}_{1}$ promotes invasion in colon carcinoma by the upregulation of MMP-2. Also, $\mathrm{DDR}_{2}$ overexpression has been found to stimulate invasion in different cancer cell lines like metastatic melanoma [38], breast [39], and prostate [40]. Given the above molecular mechanisms of DDRs among different carcinomas, the hypothesis is that DDR overexpression has the potential to connect with an unfavorable prognosis in cancer patients, which provides support for the clinical value of DDRs.

We aimed to explore the association between DDR expression levels and the prognosis of human malignant tumors in the present comprehensive meta-analysis. We pooled a total of 20 independent studies with $2,602 \mathrm{ma}$ lignant patients. Our meta-data indicated that high DDR expression was an indicator for progressive disease and poor prognosis with statistical significance for OS (HR = $1.304,95 \%$ CI $1.007-1.69, p=0.04)$. This result shows the role of DDRs overexpression as a prognostic biomarker in cancers. In subgroup analysis based on cancer types, high DDR expression was correlated with poor OS in GI cancers and UC. The reasons for this link may be existing high fibrotic conditions with high expression and crosslinking of collagens and increased interactions between collagens and DDRs in tumor stroma. Herein, different studies have shown that DDRs can promote tissue fibrosis [41-43]. More research studies are required to confirm this relationship between DDR expression and GI and UC. Also in subgroup analysis, high expression of DDRs was associated with poor survival in the Asian population. Moreover, subgroup analysis according to the $\mathrm{DDR}$ isoform showed that $\mathrm{DDR}_{2}$, but not $\mathrm{DDR}_{1}$, was correlated with poor OS in patients. This result demonstrated that $\mathrm{DDR}_{2}$ is a better prognostic marker for malignant tumors. Likewise, the clinicopathologic analyses revealed that high expression of DDRs was associated with gender; however, no prominent correlation was observed between DDR expression and TNM stage, tumor size, LNM, and age.

Our meta-analysis has some limitations that need to be pointed out. First, the majority of clinical studies carried 
out in China develop the risk of geographic bias. Second, all involved studies were only published in English, which increases selection bias. Third, existing heterogeneity among studies affects the results of the study; however, we make subgroup analyses to explore the potential sources of heterogeneity. Fourth, no appropriate and standard cutoff value was reported to make an accurate evaluation of the association between DDR expression and the survival outcome.

\section{Conclusion}

In conclusion, the present meta-analysis indicates a significant association of DDR overexpression with poor OS in several different cancers. Our findings provide further supportive evidence that DDR overexpression may be a promising potential biomarker to predict poor prognosis in cancer patients. More clinical studies are needed to clarify this association.

\section{Acknowledgements}

We thank Shahrekord University of Medical Science to provide conditions for access to databases.

\section{Conflict of Interest Statement}

The authors have no conflicts of interest to disclose.

\section{Funding Sources}

This study is supported by grants awarded by Shahrekord University of Medical Sciences (Grant No. 5595).

\section{Author Contributions}

M.-H.A. and M.S.S.A. researched the literature and conceived the study, A.F. consulted us about the field, S.S. researched the literature and collected data as the third author, and G.A.F. reviewed and edited the manuscript. M.-H.A. designed the study, wrote the first draft, and approved the final version of the manuscript.

\section{References}

1 Siegel RL, Miller KD, Jemal A. Cancer statistics, 2019. CA Cancer J Clin. 2019;69:7-34.

2 Mackillop WJ. The importance of prognosis in cancer medicine. TNM Online; 2003.

3 Miller KD, Goding Sauer A, Ortiz AP, Fedewa SA, Pinheiro PS, Tortolero-Luna G, et al. Cancer statistics for hispanics/latinos, 2018. CA Cancer J Clin. 2018;68:425-45.

4 Hur H, Ham IH, Lee D, Jin H, Aguilera KY, Oh HJ, et al. Discoidin domain receptor $1 \mathrm{ac}-$ tivity drives an aggressive phenotype in gastric carcinoma. BMC Cancer. 2017;17:87.

5 Fan Y, Xu Z, Fan J, Huang L, Ye M, Shi K, et al. Prognostic significance of discoidin domain receptor 2 (DDR2) expression in ovarian cancer. Am J Transl Res. 2016;8:2845.

6 Miao L, Zhu S, Wang Y, Li Y, Ding J, Dai J, et al. Discoidin domain receptor 1 is associated with poor prognosis of non-small cell lung cancer and promotes cell invasion via epithelial-to-mesenchymal transition. Med Oncol. 2013;30:626.

7 Ramalho S, Andrade LAA, Filho CC, Natal RA, Pavanello M, Ferracini AC, et al. Role of discoidin domain receptor 2 (DDR2) and microRNA-182 in survival of women with highgrade serous ovarian cancer. Tumour Biol. 2019;41:1010428318823988.

8 Quan J, Yahata T, Adachi S, Yoshihara K, Tanaka K. Identification of receptor tyrosine kinase, discoidin domain receptor 1 (DDR1), as a potential biomarker for serous ovarian cancer. Int J Mol Sci. 2011;12:971-82.

9 Yang SH, Baek HA, Lee HJ, Park HS, Jang KY, Kang MJ, et al. Discoidin domain receptor 1 is associated with poor prognosis of nonsmall cell lung carcinomas. Oncol Rep. 2010; 24:311-9.

10 Ford CE, Lau SK, Zhu CQ, Andersson T, Tsao MS, Vogel WF. Expression and mutation analysis of the discoidin domain receptors 1 and 2 in non-small cell lung carcinoma. $\mathrm{Br} \mathrm{J}$ Cancer. 2007;96:808-14.

11 Huo Y, Yang M, Liu W, Yang J, Fu X, Liu D, et al. High expression of DDR1 is associated with the poor prognosis in Chinese patients with pancreatic ductal adenocarcinoma. J Exp Clin Cancer Res. 2015;34:88.

12 Toy KA, Valiathan RR, Núñez F, Kidwell KM, Gonzalez ME, Fridman R, et al. Tyrosine kinase discoidin domain receptors DDR1 and DDR2 are coordinately deregulated in triplenegative breast cancer. Breast Cancer Res Treat. 2015;150:9-18.

13 Ren T, Zhang J, Zhang J, Liu X, Yao L. Increased expression of discoidin domain receptor 2 (DDR2): a novel independent prognostic marker of worse outcome in breast cancer patients. Med Oncol. 2013;30:397.

14 Stang A. Critical evaluation of the NewcastleOttawa scale for the assessment of the quality of nonrandomized studies in meta-analyses. Eur J Epidemiol. 2010;25:603-5.

15 Parmar MK, Torri V, Stewart L. Extracting summary statistics to perform meta-analyses of the published literature for survival endpoints. Stat Med. 1998;17:2815-34.

16 Wang Z, Sun X, Bao Y, Mo J, Du H, Hu J, et al. E2F1 silencing inhibits migration and invasion of osteosarcoma cells via regulating
DDR1 expression. Int J Oncol. 2017;51:163950.

17 Li D, Yang Z, Liu Z, Zou Q, Yuan Y. DDR2 and IFITM1 are prognostic markers in gallbladder squamous cell/adenosquamous carcinomas and adenocarcinomas. Pathol Oncol Res. 2019;25(1):157-67.

18 Song J, Chen X, Bai J, Liu Q, Li H, Xie J, et al. Discoidin domain receptor 1 (DDR1), a promising biomarker, induces epithelial to mesenchymal transition in renal cancer cells. Tumour Biol. 2016;37:11509-21.

19 Tsai MC, Li WM, Huang CN, Ke HL, Li CC, Yeh HC, et al. DDR2 overexpression in urothelial carcinoma indicates an unfavorable prognosis: a large cohort study. Oncotarget. 2016;7:78918.

20 Xie B, Lin W, Ye J, Wang X, Zhang B, Xiong $\mathrm{S}$, et al. DDR2 facilitates hepatocellular carcinoma invasion and metastasis via activating ERK signaling and stabilizing SNAIL1. J Exp Clin Cancer Res. 2015;34:101.

21 Lin Y, Jin H, Wu X, Jian Z, Zou X, Huang J, et al. The cross-talk between DDR1 and STAT3 promotes the development of hepatocellular carcinoma. Aging. 2020;12:14391.

22 Sugimoto K, Ito T, Woo J, Tully E, Sato K Orita $\mathrm{H}$, et al. Prognostic impact of phosphorylated discoidin domain receptor-1 in esophageal cancer. J Surg Res. 2019;235:479-86.

23 Sasaki S, Ueda M, Iguchi T, Kaneko M, Nakayama $\mathrm{H}$, Watanabe T, et al. DDR2 expression is associated with a high frequency of peritoneal dissemination and poor prognosis in colorectal cancer. Anticancer Res. 2017;37:2587-91. 
24 Velmurugan BK, Chang WH, Chung CM, Yeh CM, Lee CH, Yeh KT, et al. DDR2 overexpression in oral squamous cell carcinoma is associated to lymph node metastasis. Cancer Biomark. 2018;22:747-53.

25 Valiathan RR, Marco M, Leitinger B, Kleer CG, Fridman R. Discoidin domain receptor tyrosine kinases: new players in cancer progression. Cancer Metastasis Rev. 2012;31: 295-321.

26 Leitinger B, Kwan AP. The discoidin domain receptor DDR2 is a receptor for type $\mathrm{X}$ collagen. Matrix Biol. 2006;25:355-64.

27 Dengjel J, Akimov V, Olsen JV, Bunkenborg J, Mann M, Blagoev B, et al. Quantitative proteomic assessment of very early cellular signaling events. Nat Biotechnol. 2007;25:566-8.

28 Pertierra LR, Hughes KA, Vega GC, OlallaTárraga MÁ. High resolution spatial mapping of human footprint across Antarctica and its implications for the strategic conservation of avifauna. PLoS One. 2017;12:e0168280.

29 Rudra-Ganguly N, Lowe C, Mattie M, Chang MS, Satpayev D, Verlinsky A, et al. Discoidin domain receptor 1 contributes to tumorigenesis through modulation of TGFBI expression. PLoS One. 2014;9:e111515.

30 Kim HG, Hwang SY, Aaronson SA, Mandinova A, Lee SW. DDR1 receptor tyrosine kinase promotes prosurvival pathway through Notch1 activation. J Biol Chem. 2011;286: 17672-81.
31 Hammerman PS, Sos ML, Ramos AH, Xu C, Dutt A, Zhou W, et al. Mutations in the DDR2 kinase gene identify a novel therapeutic target in squamous cell lung cancer. Cancer Discov. 2011;1:78-89.

32 Han JA, Kim JY, Kim JI. Analysis of gene expression in cyclooxygenase-2-overexpressed human osteosarcoma cell lines. Genomics Inform. 2014;12:247.

33 Maeyama M, Koga H, Selvendiran K, Yanagimoto C, Hanada S, Taniguchi E, et al. Switching in discoid domain receptor expressions in SLUG-induced epithelial-mesenchymal transition. Cancer. 2008;113:2823-31.

34 Park HS, Kim KR, Lee HJ, Choi HN, Kim DK, Kim BT, et al. Overexpression of discoidin domain receptor 1 increases the migration and invasion of hepatocellular carcinoma cells in association with matrix metalloproteinase. Oncol Rep. 2007;18:1435-41.

35 Hansen C, Greengard P, Nairn AC, Andersson T, Vogel WF. Phosphorylation of DARPP-32 regulates breast cancer cell migration downstream of the receptor tyrosine kinase DDR1. Exp Cell Res. 2006;312:4011-8.

36 Ram R, Lorente G, Nikolich K, Urfer R, Foehr E, Nagavarapu U. Discoidin domain receptor-1a (DDR1a) promotes glioma cell invasion and adhesion in association with matrix metalloproteinase-2. J Neurooncol. 2006;76: $239-48$.
37 Hu Y, Liu J, Jiang B, Chen J, Fu Z, Bai F, et al. MiR-199a-5p loss up-regulated DDR1 aggravated colorectal cancer by activating epithelial-to-mesenchymal transition related signaling. Dig Dis Sci. 2014;59:2163-72.

38 Poudel B, Lee YM, Kim DK. DDR2 inhibition reduces migration and invasion of murine metastatic melanoma cells by suppressing MMP2/9 expression through ERK/NF- $\kappa B$ pathway. Acta Biochim Biophys Sin. 2015;47: 292-8.

39 Zhang L. The distillery.

40 Yan Z, Jin S, Wei Z, Huilian H, Zhanhai Y, Yue $\mathrm{T}$, et al. Discoidin domain receptor $2 \mathrm{fa}$ cilitates prostate cancer bone metastasis via regulating parathyroid hormone-related protein. Biochim Biophys Acta. 2014;1842:135063.

41 Coelho NM, McCulloch CA. Mechanical signaling through the discoidin domain receptor 1 plays a central role in tissue fibrosis. Cell Adh Migr. 2018;12:348-62.

42 Avivi-Green C, Singal M, Vogel WF. Discoidin domain receptor 1-deficient mice are resistant to bleomycin-induced lung fibrosis. Am J Respir Crit Care Med. 2006;174:420-7.

43 Zhang XH, Yan M, Liu L, Wu TJ, Ma LL, Wang LX. Expression of discoidin domain receptors (DDR2) in alcoholic liver fibrosis in rats. Arch Med Res. 2010;41:586-92. 